\title{
New Developments and State-of-the art AIM IR-modules
}

Wendler, Joachim

AIM INFRAROT-MODULE GmbH

Heilbronn (Germany)

\section{New Developments and State-of-the art AIM IR-modules}

\author{
J. Wendler, H. Lutz, R. Breiter, D. Eich, K. Hofmann, S. Rutzinger, R. Wollrab, J. Ziegler
}

AIM INFRAROT-MODULE GmbH, Theresienstr. 2, 74072 Heilbronn, Germany

\begin{abstract}
The production processes for IR-detectors of the 2. generation are matured. The electro optical performance parameters have reached a very high level, and constant performance values of the detectors can be expected over a long period of time.

For state of the art $480 \times 6$ linear detectors, sensitive in the $7-12 \mu \mathrm{m}$ wavelength range, AIM has demonstrated long term stability of gain correction coefficients. Driven by the development of the process parameters required for the production of these most challenging modules, AIM has started the production of 2 dimensional full format IR-modules. While $640 \times 512$ arrays with $24 \mu \mathrm{m}$ pitch sensitive in the $3-5 \mu \mathrm{m}$ (MWIR) wavelength range are routine, modules with a reduced pixel pitch of $15 \mu \mathrm{m}$ are presently moving into production at the Heilbronn facility.

This paper describes the developments and presents test results from $640 \times 512$ arrays with reduced $15 \mu \mathrm{m}$ pixel pitch as well from $3-5 \mu \mathrm{m}$ as from $7.5-10 \mu \mathrm{m}$ (LWIR) detectors.
\end{abstract}

\section{Keywords}

IR-modules, HgCdTe, $640 \times 512,15 \mu \mathrm{m}$ pitch, FPA

\section{Introduction}

During the recent years the development of state of the art 2. gen. IR-modules was driven by the requirements for limiting electro optical performance parameters as well as by the request that these performance parameters should be constant over a long time period[1]. The processes used for the production of linear $7-12 \mu \mathrm{m} 2$. generation devices are proven to fulfil these requirements. A similar development was finished in the production of $640 \times 512$ and $384 \times 288$ detectors with $24 \mu \mathrm{m}$ pixel pitch.

In 2007 AIM started the development of 2-dim. $\mathrm{HgCdTe}-\mathrm{IR}$ - detectors with $15 \mu \mathrm{m}$ pixel pitch. This class of IR-detectors takes into account the rising requests for low weight, compact, and cost-efficient IRmodules with moderate electrical power consumption but still outstanding performance parameters. 
This new detector generation is available at AIM. Due to the operational superiority and AIMs long production experience in the LWIR, this challenging spectral band was co-developed with MWIR FPAs. Test results of $640 \times 512$ detectors are presented for both spectral bands. Other detector configurations like $384 \times 288$ or $1280 \times 1024$ will follow soon in the $15 \mu \mathrm{m}$ pitch. In addition to LWIR and MWIR short wave modules are done with cut of ranging up to $2.5 \mu \mathrm{m}$, taking advantage of the specific flexibility of the MCT material system.

\section{Second Gen. Linear Arrays}

For the image quality IR-detectors two of the most critical performance parameters are the stability of the signal output voltage level, and the stability of the gain coefficients .

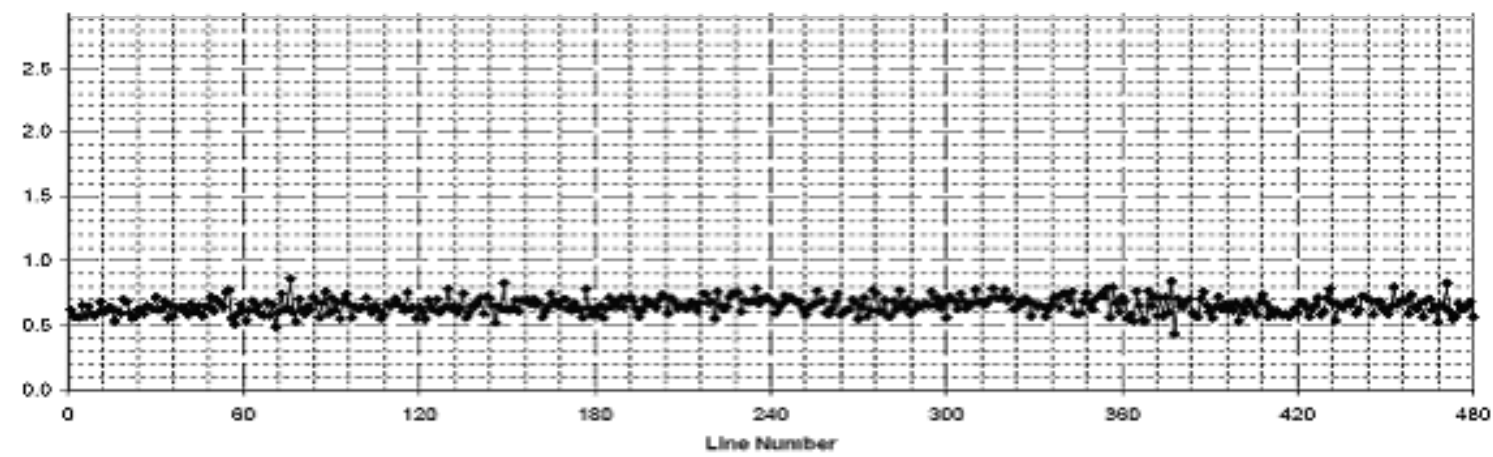

Figure 1: Stability of the signal output voltage level (linear $480 \times 6 \mathrm{HgCdTe} 7-12 \mu \mathrm{m}$ IDCA)

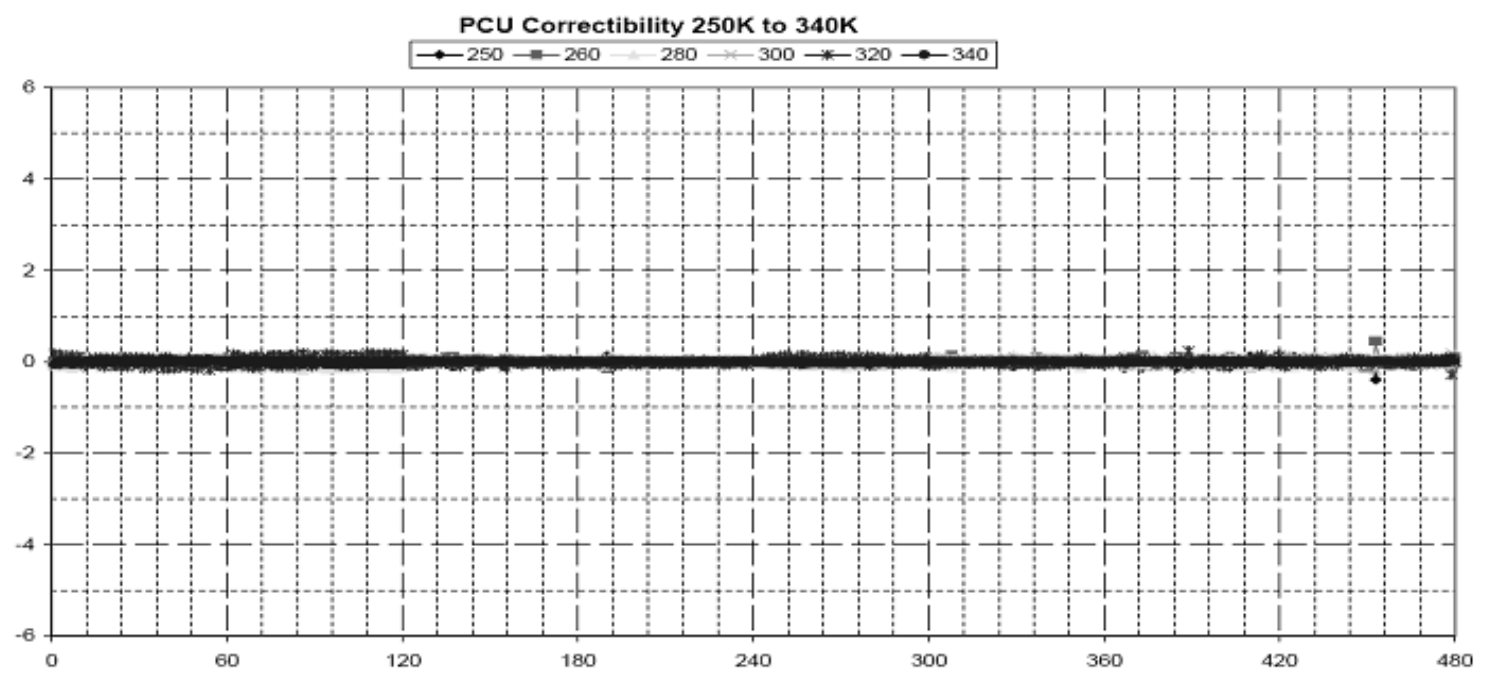

Figure 2: Stability of the gain coefficients (linear $480 \times 6 \mathrm{HgCdTe} 7-12 \mu \mathrm{m} \mathrm{IDCA}$ )

As illustrated in Figure 1, the maximum signal output voltage drift with respect to the RMS noise of each of the detector lines is less than 1 , indicating a neglectible $1 / f-$ noise contribution within the measurement time interval of 10 s.

The stability of the gain coefficients can be derived from Figure 2. In this Figure, the difference for each output signal line between the measured signal output voltage level and the voltage level calculated using the gain coefficients obtained from a previous measurement is shown in units of the RMS noise. The fixed 
pattern noise obtained after correction thus equals less than $50 \%$ of the temporal noise contribution. It should be taken into account, that the determination of the gain coefficients was made a couple of cool downs cycles before the test, and only a new scene based offset correction was done. The differences are shown for representative scene temperatures with a wide temperature range of $90 \mathrm{~K}$.

\section{3. $640 \times 512 \mathrm{HgCdTe}$-Arrays with $15 \mu \mathrm{m}$ pixel pitch}

Using such mature production technologies as developed for the state of the art linear 2. generation 7 $12 \mu \mathrm{m}$ IR-detectors and $2 \mathrm{dim}$ arrays with $24 \mu \mathrm{m}$ pitch, AIM started the $15 \mu \mathrm{m}$ pitch arrays.

The HgCdTe $640 \times 512$ Focal Plane Array (FPA) is nearly of the same size as the $384 \times 288$ FPA with $24 \mu \mathrm{m}$ pitch. This allows the assembly of such detectors in compact dewars with small cooling engines.

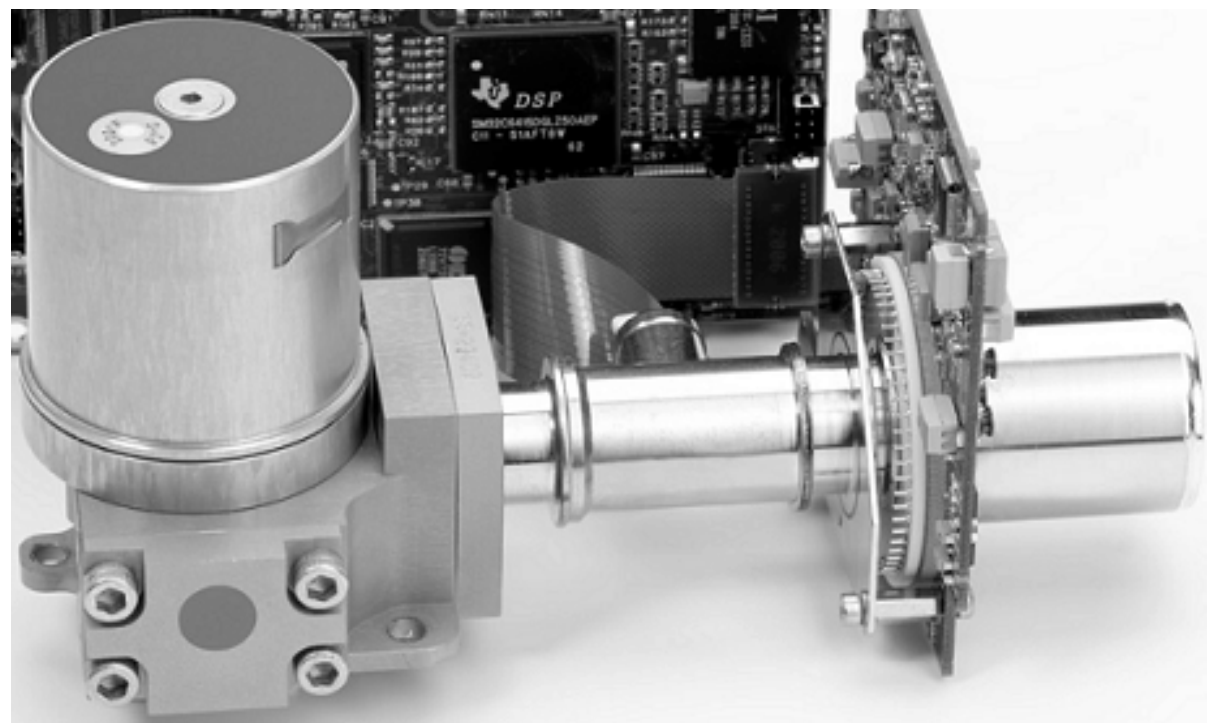

Figure 3: Standard detector module with 640x512 MWIR or LWIR FPA, CCE, and image processor

A typical application example is shown in Figure 3 . The compact imaging module consists of a $640 \times 512$ FPA with integral rotary cooler, Command and Control Electronics (CCE), and image processing board. The package equals as well the dimensional as well as the power requirements of the 384 quarter format FPA's currently used[2,3]. AIM is pushing such packages with digital corrected output since many years.

The electro optical performance parameters obtained with the new generation of staring FPA's are listed in Table 1. Figure 4 shows examples of images obtained from a MWIR FPA on the left side and a LWIR FPA on the right. The images prove the excellent spatial resolution expected from a $640 \times 512$ device as well as the thermal resolution. Both detectors provide a homogenious image corrected for a wide range of background temperature. The number of defect pixels required to be replaced is very low for the MWIR FPA, and also low for the LWIR detector.

\begin{tabular}{|l|c|c|}
\hline Performance Parameter & MWIR & LWIR \\
\hline typical NETD (300K at half well) & $25 \mathrm{mK}$ & $36 \mathrm{mK}$ \\
\hline typical IETD & $<$ NETD & $<$ NETD \\
\hline typical Quantum Efficiency & $80 \%$ & $70 \%$ \\
\hline typical no. of Defective Pixels & $<0.5 \%$ & $<1 \%$ \\
\hline
\end{tabular}

Table 1: Performance parameters of $640 \times 512 \mathrm{HgCdTe}$ staring FPA detectors with $15 \mu \mathrm{m}$ pixel pitch 
MWIR 640x512 FPA 15 $\mu \mathrm{m}$ pitch

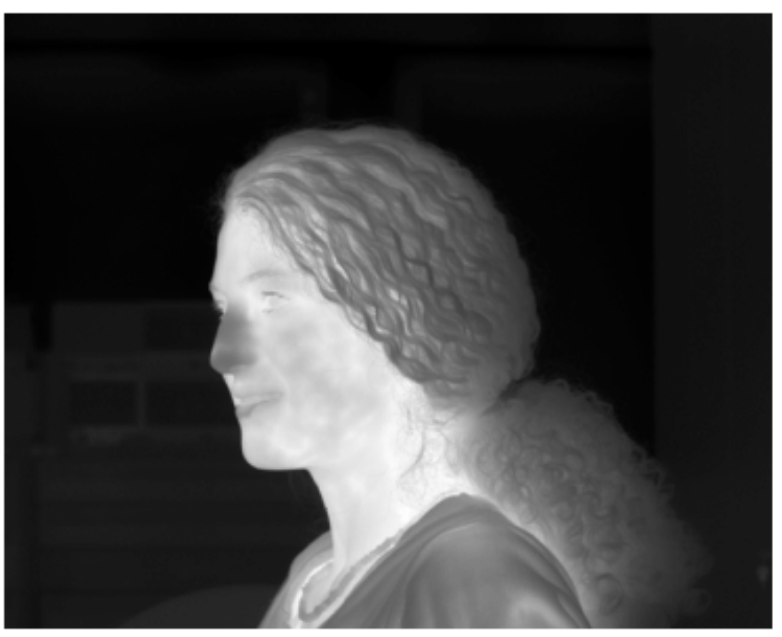

LWIR 640x512 FPA 15 $\mu \mathrm{m}$ pitch

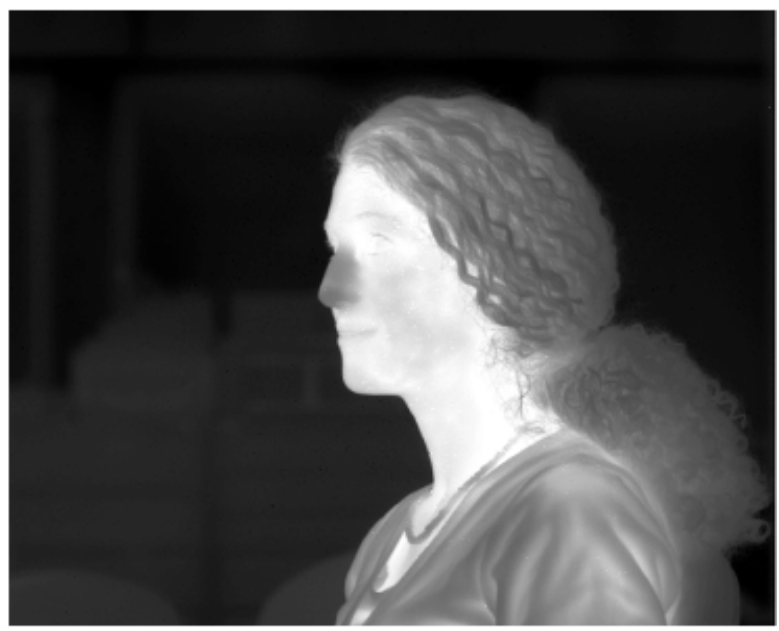

Figure 4: Examples of $640 \times 512 \mathrm{HgCdTe}$ staring FPA $15 \mu$ pitch detector images

\section{Detector Technology}

AIMs state of the art FPA technology applies a mature soldering process for the hybridization, improved the stability of the detector ROIC (Read Out Integrated Circuit) compounds by use of an underfiller $\mathrm{n}$ and removal of the IR-detector substrate Temperature cycle stability was proven for more than $10000 \mathrm{cool}$ down cycles without namable degradation of the electro optical performance or increase of pixel outages. Figure 5 illustrates this process.

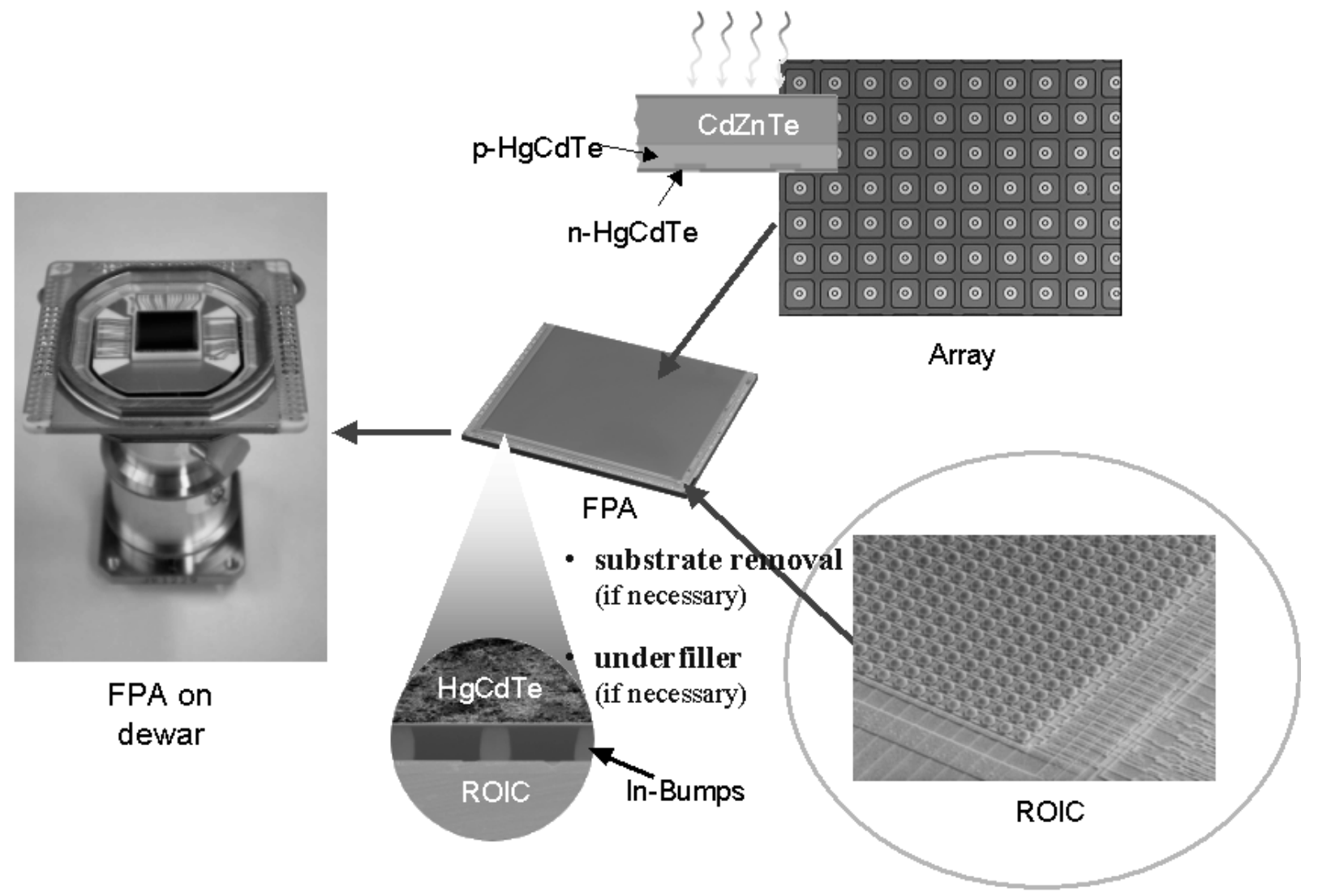

Figure 5: Illustration of FPA production process 
The IR-detector technology is also modified to match the demanding requirements for the staring FPA's under high illumination conditions. The ohmic resistance in series with the detector diodes in the substrate path was significantly reduced to minimize the photocurrent induced shift of the bias voltage applied to the photodiode. Figure 6 shows an example of performance improvement acquired by this technology.

The ROIC's developed for the new detectors show additional features such as the choice between IWR and ITR (Integrate While Read; Integrate Then Read) read out modes, independent inversion of both scan directions, or selectable subframe windowing in fine steps of 1 pixel vertical and 4 pixel horizontal. Depending upon the required frame rate, the number of detector signal outputs is selectable. While for standard $50 \mathrm{~Hz}$ applications 2 analog output signal channel are sufficient, for high frame rates of $100 \mathrm{~Hz}$ the ROIC can be switched to a 4 output channel operating mode.

For the simplified control of all ROIC functions such as the select of the integration time, the devices have a serial data interface allowing the programming of the ROIC internal control registers for the set up of the desired operating modes.
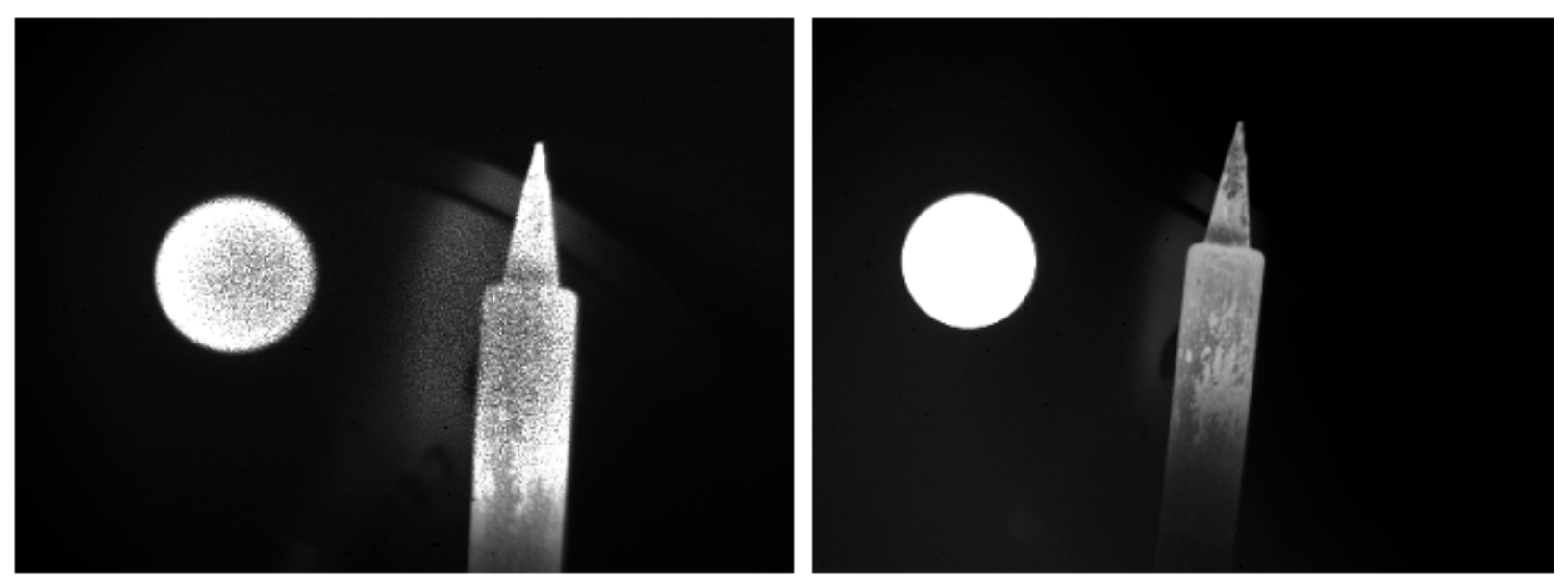

Figure 6: Both images show a hot soldering iron and the aperture of a high temperature black body at $500^{\circ} \mathrm{C}$. While the standard MWIR FPA image on the left size provides the characteristic artifacts caused by the self debiasing effects, the right image of the improved MWIR FPA neither shows the typical intensity reductions in the middle of the black body aperture nor the apparent increase in aperture diameter. The detailed structures of the hot soldering iron surface on the right image are also free of any decrease in intensity as visible in the left image.

\section{Conclusions}

AIM manufactures large LWIR 2. generation IR-detectors for some of the most challenging international programs. These detectors provide stable electro optical performance parameters over a long time period and a large number of cool down cycles.

For new programs with requirements for for low weight, compact and cost-efficient IR-modules, a new family of FPA's with $15 \mu \mathrm{m}$ pitch was developed. The $640 \times 512$ arrays are available now with excellent performance in the MWIR and more important also LWIR. Further detectors with $384 \times 288$ and $1280 \times 1024$ pixels will follow. 


\section{Acknowledgement}

The authors would like to thank the AIM teams designing and producing high performance products with excellent reliability.

\section{References}

1. J. Wendler, W. Cabanski, I. Rühlich, J. Ziegler, "High-performance IR detector modules", Vol.5251, Proceedings SPIE Detectors and Associated Signal Processing, 2003

2. R. Breiter, W. Cabanski, T. Ihle, K.-H. Mauk, W. Rode, "AIM thermal imagers for reconnaissance and targeting applications", Vol. 6206, Proceedings SPIE Defence\&Security, 2006

3. R. Breiter, W. Cabanski, T. Ihle, K.-H. Mauk, W. Rode, "HuntIR thermal imagers for reconnaissance and targeting applications", Vol. 5406, Proceedings SPIE Defence\&Security, 2004 